\title{
$\mathrm{HSC} / 17 / 01$
}

\section{Variance stabilizing transformations for electricity spot price forecasting}

\author{
Bartosz Uniejewski ${ }^{1}$ \\ Rafał Weron ${ }^{2}$ \\ Florian Ziel ${ }^{3}$
}

10

${ }^{1}$ Faculty of Pure and Applied Mathematics, Wrocław

University of Technology, Poland

2 Department of Operations Research, Wrocław University of Technology, Poland

3 Faculty of Business Administration and Economics, Universität Duisburg-Essen, Germany 


\title{
Variance Stabilizing Transformations for Electricity Spot Price Forecasting
}

\author{
Bartosz Uniejewski, Rafał Weron and Florian Ziel
}

\begin{abstract}
Most electricity spot price series exhibit price spikes. These extreme observations may significantly impact the obtained model estimates and hence reduce efficiency of the employed predictive algorithms. For markets with only positive prices the logarithmic transform is the single most commonly used technique to reduce spike severity and consequently stabilize the variance. However, for datasets with very close to zero (like the Spanish) or negative (like the German) prices the log-transform is not feasible. What reasonable choices do we have then?

To address this issue, we conduct a comprehensive forecasting study involving 12 datasets from diverse power markets and evaluate 16 variance stabilizing transformations. We find that the probability integral transform (PIT) combined with the standard Gaussian distribution yields the best approach, significantly better than many of the considered alternatives.
\end{abstract}

Index Terms-Electricity spot price, Forecasting, Variance stabilizing transformation, Probability integral transform, Price spike, Diebold-Mariano test

\section{INTRODUCTION}

A critical issue in the calibration of electricity price forecasting models is their sensitivity to price spikes [1]. The latter are one of the most pronounced features of deregulated power markets and nearly all spot price time series exhibit them. Spikes come in all sorts and sizes, mostly positive but in some markets also negative [2], [3]. A statistically appropriate modeling framework would require a dedicated treatment of these 'outliers', either via robust estimation algorithms [4] or models with explicit spike components [5]-[7]. However, robust techniques are not popular in the electricity price forecasting (EPF) literature and most studies utilize Ordinary Least Squares (OLS) methods, while non-linear models generally do not outperform linear ones in short-term EPF [1], [8].

As a working remedy some authors suggest filtering electricity prices with a 'reasonable' procedure for outlier detection, then calibrating the model to spike-filtered data, with the spikes replaced by more 'normal' values [3], [9]-[11]. Others advocate transforming the original data, running a model on transformed prices, then applying the inverse transformation to obtain forecasts [12], [13]. For markets with only positive prices, the logarithm is the single most commonly used transform to reduce spike severity and consequently stabilize the variance [1]. However, with the increased market

The study was partially supported by the National Science Center (NCN, Poland) through grant no. 2015/17/B/HS4/00334 (to BU and RW).

BU is with the Faculty of Pure and Applied Mathematics and RW is with the Department of Operations Research, Wrocław University of Science and Technology, 50-370 Wrocław, Poland. FZ is with the Faculty of Business Administration and Economics, Universität Duisburg-Essen, D-45141 Essen, Germany. E-mails: uniejewskibartosz@gmail.com, rafal.weron@pwr.edu.pl, Florian.Ziel@uni-due.de. penetration of renewable energy, the price series recorded nowadays quite often include very close to zero or negative values. Obviously, the log-transform is not a feasible option then. Somewhat surprisingly, not too many viable alternatives have been considered in the EPF literature so far. With this study we want to fill the gap and conduct a comprehensive forecasting study involving datasets from 12 power markets and evaluate 16 variance stabilizing transformations (VSTs), ranging from simple threshold-type cutoffs, via generalized Box-Cox type transforms to the probability integral transform (PIT) based approaches.

The paper is structured as follows. In Section II we briefly describe the datasets, then in Section III define the notation, the basic forecasting model and discuss the 16 VSTs considered in this study. In Section IV we evaluate their performance across the 12 datasets. We also test for statistically significant differences in their forecasting performance using two variants of the Diebold-Mariano [14] test, thus provide robust guidelines to preprocessing electricity spot prices prior to fitting time series or computational intelligence models. Finally, in Section V we wrap up the results and conclude.

\section{DATASETS}

We consider a total of twelve electricity spot price datasets, see Table I. Eleven originate from six major European power markets, including the European Power Exchange (EPEX SPOT) for power trading in Germany, France, Austria, Switzerland and Luxembourg, the Nordic power exchange Nord Pool and the Iberian OMIE (Spain and Portugal). All eleven include day-ahead prices (quoted in EUR/MWh) at hourly resolution and cover a six year period from $30 \mathrm{Jul}$ 2010 to 28 Jul 2016. The 12th dataset comes from the price track of the Global Energy Forecasting Competition 2014 (GEFCom2014) and includes locational marginal prices (LMPs, i.e., zonal prices; quoted in USD/MWh) at an hourly resolution from 1 Jan 2011 to 17 Dec 2013 [8]. The exact origin of the data has never been revealed by the organizers but - given its features - it quite likely comes from one of the U.S. markets. Note, that like Uniejewski et al. [15], we use the terms spot and day-ahead interchangeably, which is line with the majority of literature on European electricity markets. However, in the U.S., the 'spot' is rather used to refer to the real-time market, while the day-ahead market is usually called the forward market [16], [17].

Furthermore, because of the clock changes to and from the daylight saving time, we have to do minor adjustments to the data to obtain well defined price processes. For the European 
TABLE I

THE TWELVE CONSIDERED ELECTRICITY SPOT PRICE SERIES.

\begin{tabular}{lll}
\hline Electricity market \& region & Acronym & Source \\
\hline \multicolumn{1}{c}{ 6-year period (30 Jul 2010 to 28 Jul 2016) } \\
BELPEX, Belgium & BELPEX.BE & belpex.be \\
EPEX, Switzerland & EPEX.CH & epexspot.com \\
EPEX, Germany \& Austria & EPEX.DE+AT & epexspot.com \\
EPEX, France & EPEX.FR & epexspot.com \\
EXAA, Germany \& Austria & EXAA.DE+AT & exaa.at \\
Nord Pool, West Denmark & NP.DK1 & nordpoolspot.com \\
Nord Pool, East Denmark & NP.DK2 & nordpoolspot.com \\
Nord Pool, System price & NP.SYS & nordpoolspot.com \\
OMIE, Spain & OMIE.ES & omie.es \\
OMIE, Portugal & OMIE.PT & omie.es \\
OTE, Czechia & OTE.CZ & ote-cr.cz \\
\hline \multicolumn{3}{c}{ 3-year period (1 Jan 2011 to 17 Dec 2013) } \\
GEFCom2014 competition & GEFCom2014 & Ref. [8] \\
\hline
\end{tabular}

data we interpolate the missing hour in March and average the doubled hour in October. The GEFCom2014 data was released clock-change adjusted [8].

\section{MODELS}

First, let us fix the notation. We denote by $P_{d, h}$ the electricity price in the day-ahead market for day $d$ and hour $h$ and by $Y_{d, h}$ the transformed data, i.e., $Y_{d, h}=f\left(P_{d, h}\right)$, where $f(\cdot)$ is a given variance stabilizing transformation. After computing the forecasts, we apply the inverse transformation to obtain the electricity spot price forecasts, i.e., $\hat{P}_{d, h}=f^{-1}\left(\hat{Y}_{d, h}\right)$. Naturally, we evaluate the accuracy of the considered methods using $\hat{P}_{d, h}$, not the forecasts of the transformed series.

Moreover, most transformations we consider work on "normalized' prices: $p_{d, h}=\frac{1}{b}\left(P_{d, h}-a\right)$, where $a$ is the shift and $b$ is the scale. Later in the text we report results for two sets of 'normalizing' parameters:

1) set $_{1}:(a, b)=$ (median, MAD), i.e., $a$ is the median of $P_{d, h}$ in the 730-day calibration sample and $b$ is the sample median absolute deviation (MAD) around the sample median adjusted by a factor for asymptotically normal consistency to the standard deviation. This factor is $\frac{1}{z_{0.75}} \approx 1.4826$ where $z_{0.75}$ is the $75 \%$ quantile of the normal distribution; in $\mathrm{R}$ this is the default option if one runs mad $(x)$, in Matlab this corresponds to $1.4826 * \operatorname{mad}(x, 1)$.

2) set $_{2}:(a, b)=$ (mean, std), i.e., $a$ is the mean and $b$ is the standard deviation of $P_{d, h}$ in the 730-day calibration sample.

Obviously, the inverse transformations listed below require inverting the 'normalization', i.e., $P_{d, h}=b \cdot p_{d, h}+a$, but for notational simplicity we leave them in terms of $p_{d, h}$.

\section{A. The basic forecasting model}

Our choice of the basic forecasting model used in this study is guided by three factors. Firstly, the existing literature on short-term EPF which has generally favored the multivariate framework, with prices for each hour of the day modeled independently by 24 parsimonious models rather than jointly by one large model [1], [18]. Secondly, the desire to perform a comprehensive study of the influence of VSTs on the forecasting performance using a state-of-the-art structure, that builds on the results of the most recent EPF studies on variable selection [13], [15]. Thirdly, computational efficiency required to produce forecasts for many VSTs and many datasets within a rolling calibration window scheme, which favors regression over neural network setups. Taking all three factors into account, we have decided to use the expert $_{\text {Dow,nl model of }}$ Ziel and Weron [13], which is a parsimonious autoregressive structure estimated using OLS, that outperformed not only 15 other expert ${ }^{1}$ models, but also much larger multi- and univariate autoregressive specifications. In this model, the transformed (or original if no transformation is used) dayahead electricity price for day $d$ and hour $h$ is given by:

$$
\begin{aligned}
Y_{d, h}= & \beta_{h, 1}+\underbrace{\beta_{h, 2} Y_{d-1, h}+\beta_{h, 3} Y_{d-2, h}+\beta_{h, 4} Y_{d-7, h}}_{\text {autoregressive terms }} \\
& +\underbrace{\beta_{h, 5} Y_{d-1}^{\min }+\beta_{h, 6} Y_{d-1}^{\max }}_{\text {non-linear effects }}+\underbrace{\beta_{h, 7} Y_{d-1,24}}_{\text {end-of-day effect }} \\
& +\underbrace{\sum_{j=1}^{7} \beta_{h, j+7} D_{j}}_{\text {weekday dummies }}+\varepsilon_{d, h},
\end{aligned}
$$

where $Y_{d-1, h}, Y_{d-2, h}$ and $Y_{d-7, h}$ are prices for the same hour yesterday, two days ago and a week ago, $Y_{d-1}^{\min }$ and $Y_{d-1}^{\max }$ are yesterday's minimum and maximum prices (they provide a non-linear link between yesterday's and today's prices), $Y_{d-1,24}$ is the price for midnight (the last known price), $D_{1}, \ldots, D_{7}$ are weekday dummies $\left(D_{1}=1\right.$ for Monday and 0 otherwise, $D_{2}=1$ for Tuesday and 0 otherwise, etc.) and $\varepsilon_{d, h}$ is the noise term (uncorrelated and with finite variance). Note, that we have also considered several other expert models from [13], [15], but they all yielded qualitatively the same results while being outperformed by the expert $_{\text {Dow,nl model. }}$

\section{B. Variance stabilizing transformations (VSTs)}

The logarithmic transform is by far the most popular approach to reducing spike severity and stabilizing the variance [20]. However, for datasets with very close to zero (like OMIE.ES and OMIE.PT) or negative electricity prices (like EPEX.DE+AT) the standard log-transform is not feasible. In this Section we discuss eight types of transformations that can be used in such a context. Some have been already utilized in $\mathrm{EPF}$, others are new. The first seven are illustrated in Fig. 1 and come in two variants denoted by a subscript, with 1 standing for set $_{1}$ and 2 for set $_{2}$ 'normalization' (see above). The last class builds on the probability integral transform (PIT) and does not require 'normalization'.

Some of the earliest suggested solutions to reducing the 'outlier effect' of electricity price spikes include limiting their severity by setting an upper limit on prices (known as clipping in signal processing [21] and winsorizing in statistics [20]) or damping all observations above a certain threshold using the logarithmic function [9]. In this study we consider both variants with the threshold set to the commonly used level of

\footnotetext{
${ }^{1}$ Since such models are built on some prior knowledge of experts, following Uniejewski et al. [15] and Ziel [19], we refer to them as expert models.
} 


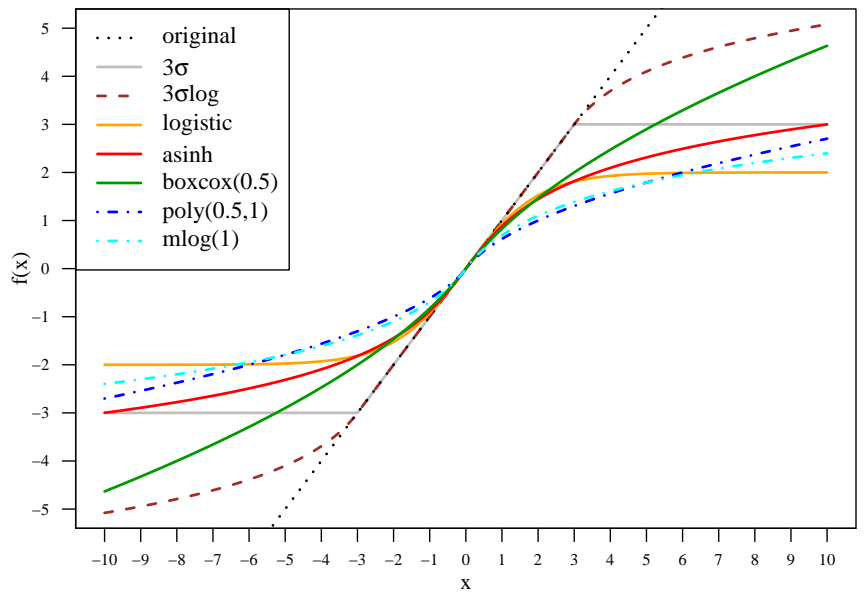

Fig. 1. Visualization of seven out of eight classes of transformations considered in this study. All are shifted and scaled so that $f(0)=0$ and the slope at $x=0$ is $45^{\circ}$ to emphasize the differences between them. The effect of applying the 8th class is depicted in Fig. 2.

$k=3$ standard deviations of the (transformed) price series in the calibration window [17]; we have also tested variants with $k=2$ and 4 , but they performed worse. The first, 'clipping' variant is denoted by $3 \sigma$ and given by:

$$
Y_{d, h}= \begin{cases}3 \operatorname{sgn}\left(p_{d, h}\right) & \text { for }\left|p_{d, h}\right|>3 \\ p_{d, h} & \text { for }\left|p_{d, h}\right| \leq 3\end{cases}
$$

with inverse $p_{d, h}=\min \left(\max \left(Y_{d, h},-3\right), 3\right)$, where $\operatorname{sgn}(x)$ is the sign of $x$. The second, 'log-damping' variant is denoted by $3 \sigma \log$ and given by:

$Y_{d, h}= \begin{cases}\operatorname{sgn}\left(p_{d, h}\right)\left\{\log \left(\left|p_{d, h}\right|-2\right)+3\right\} & \text { for }\left|p_{d, h}\right|>3, \\ p_{d, h} & \text { for }\left|p_{d, h}\right| \leq 3,\end{cases}$

with inverse:

$$
p_{d, h}= \begin{cases}\operatorname{sgn}\left(Y_{d, h}\right)\left(e^{\left|Y_{d, h}\right|-3}+2\right) & \text { for }\left|Y_{d, h}\right|>3, \\ Y_{d, h} & \text { for }\left|Y_{d, h}\right| \leq 3 .\end{cases}
$$

In Figure 1 we can clearly see that $3 \boldsymbol{\sigma}$ is not differentiable and bounded at $x= \pm 3$, whereas $\mathbf{3} \boldsymbol{\sigma} \log$ is a smooth function with $3 \sigma \log (x) \rightarrow \pm \infty$ as $x \rightarrow \pm \infty$ and a log-damping effect.

The next transformation class we consider is given by the logistic function that is a sigmoid curve and has many applications in data analytics. For instance, it is used as a link function in generalized linear models (GLM) and is a very popular choice for the activation function in neural nets [22]. However, to our best knowledge, it has never been applied as a VST in electricity price forecasting. We denote this transformation by logistic and define as:

$$
Y_{d, h}=\left(1+e^{-p_{d, h}}\right)^{-1} .
$$

Its inverse is the so-called logit function:

$$
p_{d, h}=\log \left(\frac{Y_{d, h}}{1-Y_{d, h}}\right) .
$$

As visualized in Fig. 1, the logistic transformation is bounded, like $3 \sigma$, but is a smooth function. Note, that to avoid numerical issues we clip the forecasts of $Y_{d, h}$ to the interval
$[0.001,0.999]$, i.e., replace all values outside the interval by the lower or upper bound, before applying Eqn. (6).

The third class of transformations is built around the area hyperbolic sine, i.e., the inverse of the hyperbolic sine. Interestingly, this transformation has been already used in the context of electricity prices by Schneider [12], but the article went unnoticed. Recently, Ziel and Weron [13] utilized it in an extensive empirical study on multi- and univariate EPF models, motivated by the transformation's ability to preserve unimodality of the sample density. In this paper we denote it by asinh and define as:

$$
Y_{d, h}=\operatorname{asinh}\left(p_{d, h}\right) \equiv \log \left(p_{d, h}+\sqrt{p_{d, h}^{2}+1}\right),
$$

with inverse $p_{d, h}=\sinh \left(Y_{d, h}\right)$. In Figure 1 we can clearly see the damping behavior of the logarithm in Eqn. (7), while in Fig. 2 the transformation's performance for a sample dataset.

Recall, that the log-transform is a special case of the so-called Box-Cox transform, a very popular VST in time series analysis [23]. Like the logarithm, the standard Box-Cox transform is not defined for non-positive values. However, in this study we consider a robust (to zeros and negative values) variant [24], denoted by $\operatorname{box} \operatorname{cox}(\lambda)$ and defined as:

$$
Y_{d, h}=\operatorname{sgn}\left(p_{d, h}\right) \begin{cases}\frac{\left(\left|p_{d, h}\right|+1\right)^{\lambda}-1}{\lambda} & \text { for } \lambda>0 \\ \log \left(\left|p_{d, h}\right|+1\right) & \text { for } \lambda=0\end{cases}
$$

with inverse:

$$
p_{d, h}=\operatorname{sgn}\left(Y_{d, h}\right) \begin{cases}\left(\lambda\left|Y_{d, h}\right|+1\right)^{\frac{1}{\lambda}}-1 & \text { for } \lambda>0, \\ e^{\left|Y_{d, h}\right|}-1 & \text { for } \lambda=0 .\end{cases}
$$

Obviously, the robust (to zeros and negative values) variant of the log-transform is obtained for $\lambda=0$. However, here we use $\lambda=0.5$, which was selected based on a limited optimization study. With this choice of $\lambda$, the $\operatorname{boxcox}(\lambda)$ transformation exhibits a polynomial damping effect, see Fig. 1.

Motivated by the robust Box-Cox transform, we introduce two new transforms - the polynomial and the mirror-log. We denote the former by $\operatorname{poly}(\lambda, c)$ and define as:

$$
Y_{d, h}=\operatorname{sgn}\left(p_{d, h}\right)\left[|| p_{d, h}\left|+\left(\frac{c}{\lambda}\right)^{\frac{1}{\lambda-1}}\right|^{\lambda}-\left(\frac{c}{\lambda}\right)^{\frac{\lambda}{\lambda-1}}\right],
$$

with inverse:

$p_{d, h}=\operatorname{sgn}\left(Y_{d, h}\right)\left[\left(\left|Y_{d, h}\right|+\left(\frac{c}{\lambda}\right)^{\frac{\lambda}{\lambda-1}}\right)^{\frac{1}{\lambda}}-\left(\frac{c}{\lambda}\right)^{\frac{1}{\lambda-1}}\right]$.

The $\operatorname{poly}(\lambda, c)$ transform is a two parameter family. Here we use $\lambda=0.125$ and $c=0.05$, which was selected based on a limited optimization study.

The mirror-log is a straightforward generalization of the logtransform with a mirror image of the logarithm for negative values. More precisely, with the logarithm flipped with respect to the origin from the first to the third quadrant, see Fig. 1. We denote it by $\operatorname{mog}(c)$ and define as:

$$
Y_{d, h}=\operatorname{sgn}\left(p_{d, h}\right)\left[\log \left(\left|p_{d, h}\right|+\frac{1}{c}\right)+\log (c)\right],
$$



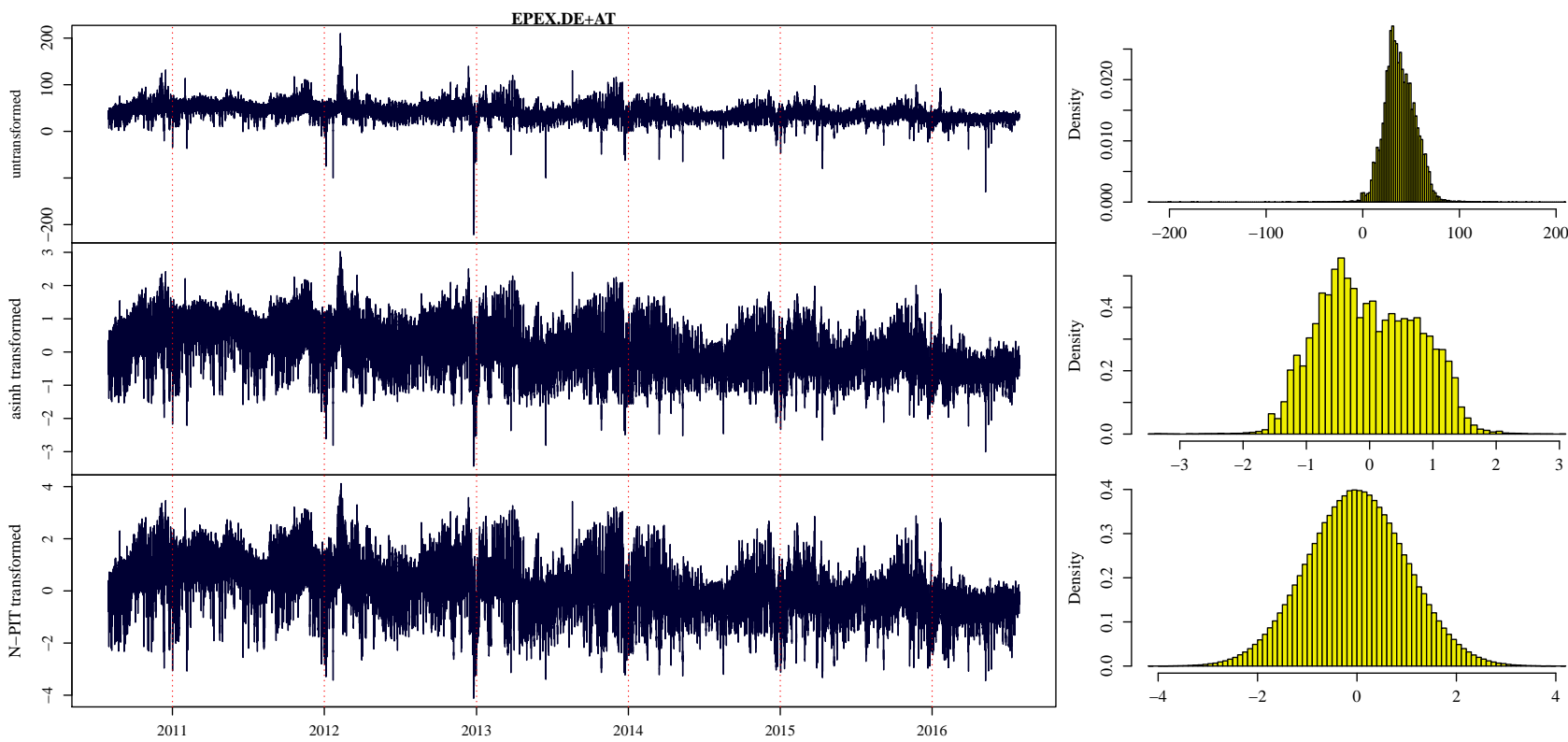

Fig. 2. Time series plot of the original (in EUR/MWh; top), $\mathbf{a s i n h}_{2}$-transformed (middle) and N-PIT-transformed EPEX.DE+AT spot prices. The marginal densities are depicted in the right panels.

with inverse:

$$
p_{d, h}=\operatorname{sgn}\left(Y_{d, h}\right)\left(e^{\left|Y_{d, h}\right|-\log c}-\frac{1}{c}\right) .
$$

The $\operatorname{mlog}(c)$ transform is a one parameter family. We use $c=$ $\frac{1}{3}$, which again was selected based on a limited optimization study. Similarly as the standard log-transform and asinh, the mirror-log exhibits a log-damping effect, see Fig. 1. Both the mlog and poly are constructed so that they have a slope of $c$ at the origin. Hence, $\operatorname{mlog}(1)$ is the same as $\operatorname{boxcox}(0)$.

The last class of transformations we consider here is based on the so-called probability integral transform: $\operatorname{PIT}(X)=$ $F_{X}(X)$, where $F_{X}$ is the cumulative distribution function (cdf) of random variable $X$. The origin of the PIT is not known, but as Gneiting et al. [25] argue, it can be traced back at least to the works of Karl Pearson in the 1930s. In the empirical context we usually do not know the true distribution of $X$, hence the PIT is rather defined as: $\operatorname{PIT}(X)=\hat{F}_{X}(X)$, where $\hat{F}_{X}$ is an estimate (e.g., empirical cdf) or a distributional forecast of $F_{X}$. If the latter is perfect, i.e., $\hat{F}_{X}=F_{X}$, then $\operatorname{PIT}(X)$ is an independent and uniformly distributed variable. This property can be used to evaluate distributional forecasts [25].

In our context, however, the following transformation will be more useful than the PIT itself:

$$
Y_{d, h}=G^{-1}\left(\operatorname{PIT}\left(P_{d, h}\right)\right)=G^{-1}\left(\hat{F}_{P_{d, h}}\left(P_{d, h}\right)\right),
$$

where $G^{-1}$ is the inverse of some continuous distribution. Note, that we apply the PIT to original prices, $P_{d, h}$, as this transformation does not require 'normalization' of the inputs. We consider two variants: (i) normal or N-PIT, with $G^{-1}$ being the inverse of the standard normal cdf, and (ii) Student- $t$ or t-PIT, with $G^{-1}$ being the inverse of the standard Student$t$ distribution with $\nu=8$ degrees of freedom (we have also tried other $\nu$ 's, but lower values yielded too heavy tails, while larger a behavior too similar to that of N-PIT).
The N-PIT transformation, misleadingly called the Nataf transformation ${ }^{2}$, has been used in [27] to 'normalize' Spanish electricity prices. However, the authors have not computed forecasts, only concluded that a 'modified Nataf' transformation (that corrected for the observed zero prices) allowed them to obtain a normal cdf of the transformed prices. The t-PIT transformation, on the other hand, has not been used in EPF before. Finally note, that the inverse of Eqn. (14) is given by:

$$
P_{d, h}=\hat{F}_{P_{d, h}}^{-1}\left(G\left(Y_{d, h}\right)\right),
$$

with $G$ being either the standard normal or Student $t$ cdf.

Since the normal distribution has exponential tails and the Student- $t$ has polynomial, the N-PIT has an exponential spike damping behavior and t-PIT a polynomial one. Hence, extreme price spikes remain larger for t-PIT-transformed data than for N-PIT-transformed. In Figure 2 we visualize the effect of applying the N-PIT compared to that of original (i.e., untransformed) and $\mathbf{a s i n h}_{2}$-transformed data. We clearly see, that the N-PIT-transformed data histogram looks pretty much like a standard normal density. In contrast, $\operatorname{asinh}_{2}$ preserves the original shape of the histogram of the untransformed data, but damps the price spikes towards the center.

\section{EMPIRICAL STUDY}

Like Ziel and Weron [13], we use a 730-day (ca. twoyear) rolling calibration window testing scheme. First, all considered models are estimated using data from the initial calibration period (i.e., from $31 \mathrm{Jul} 2010$ to $30 \mathrm{Jul} 2012$ for the European datasets and from 1 Jan 2011 to 30 Dec 2012 for GEFCom2014) and forecasts for all 24 hours of $31 \mathrm{Jul} 2012$

\footnotetext{
${ }^{2}$ The Nataf transformation is a two stage procedure, popularized in reliability engineering by [26]. First, correlated multivariate data is N-PITtransformed independently in each dimension, then the correlation is eliminated by applying the Cholesky factorization.
} 
TABLE II

MEAN ABSOLUTE ERRORS (MAE) ACROSS THE WHOLE TEST PERIOD FOR THE 12 MARKETS (IN COLUMNS) AND THE 16 VARIANCE STABILIZING TRANSFORMATIONS (VSTS; IN ROWS). A HEAT MAP IS USED TO INDICATE BETTER ( $\rightarrow$ GREEN) AND WORSE ( $\rightarrow$ RED) PERFORMING VSTS. IN THE LAST TWO COLUMNS WE REPORT THE AGGREGATE M.P.D.F.B. ERROR MEASURE, SEE EQN. (18), FOR THE MAE AND THE RMSE.

\begin{tabular}{|c|c|c|c|c|c|c|c|c|c|c|c|c|c|c|}
\hline \multirow[t]{2}{*}{ VST } & \multirow{2}{*}{ 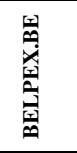 } & \multirow{2}{*}{ 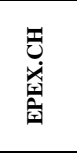 } & \multirow{2}{*}{ 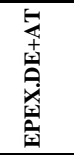 } & \multirow{2}{*}{ 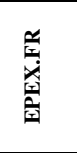 } & \multirow{2}{*}{ 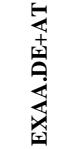 } & \multirow{2}{*}{$\begin{array}{l}\overline{\mathbf{u}} \\
\text { à }\end{array}$} & \multirow{2}{*}{ 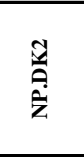 } & \multirow{2}{*}{$\begin{array}{l}\infty \\
\dot{z} \\
\dot{z}\end{array}$} & \multirow{2}{*}{ 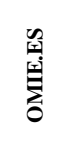 } & \multirow{2}{*}{ t) } & \multirow{2}{*}{ 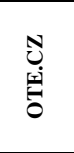 } & \multirow{2}{*}{ 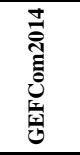 } & \multicolumn{2}{|c|}{ m.p.d.f.b. } \\
\hline & & & & & & & & & & & & & MAE & RMSE \\
\hline original & 6.379 & 4.019 & 5.370 & 5.296 & 4.282 & 6.200 & 5.186 & 1.890 & 5.825 & 5.999 & 4.670 & 7.472 & $5.22 \%$ & $9.46 \%$ \\
\hline $3 \sigma_{1}$ & 6.069 & 3.991 & 5.180 & 4.866 & 4.249 & 5.269 & 4.948 & 1.834 & 6.157 & 6.326 & 4.592 & 8.920 & $3.98 \%$ & $6.80 \%$ \\
\hline $3 \sigma_{2}$ & 6.088 & 3.989 & 5.199 & 4.880 & 4.257 & 5.379 & 5.005 & 1.830 & 5.858 & 6.013 & 4.599 & 7.720 & $2.04 \%$ & $3.04 \%$ \\
\hline $3 \sigma \log _{1}$ & 6.114 & 3.993 & 5.197 & 4.882 & 4.270 & 5.331 & 5.004 & 1.829 & 6.043 & 6.325 & 4.605 & 7.591 & $2.59 \%$ & $3.78 \%$ \\
\hline $3 \sigma \log _{2}$ & 6.137 & 3.993 & 5.221 & 4.905 & 4.273 & 5.434 & 5.055 & 1.838 & 5.865 & 6.055 & 4.611 & 7.517 & $2.29 \%$ & $1.94 \%$ \\
\hline $\operatorname{logistic}_{1}$ & 6.138 & 4.133 & 5.369 & 5.149 & 4.486 & 5.296 & 4.971 & 1.980 & 6.817 & 6.918 & 4.740 & 8.042 & $7.38 \%$ & $14.29 \%$ \\
\hline $\operatorname{logistic}_{2}$ & 6.047 & 4.126 & 5.250 & 4.946 & 4.422 & 5.303 & 4.928 & 1.908 & 6.578 & 6.721 & 4.639 & 7.921 & $5.24 \%$ & $10.81 \%$ \\
\hline $\operatorname{asinh}_{1}$ & 6.064 & 4.074 & 5.226 & 4.999 & 4.289 & 5.197 & 4.884 & 1.808 & 6.134 & 6.290 & 4.601 & 7.022 & $1.85 \%$ & $2.73 \%$ \\
\hline $\operatorname{asinh}_{2}$ & 6.057 & 4.080 & 5.207 & 4.949 & 4.288 & 5.317 & 4.920 & 1.803 & 6.018 & 6.168 & 4.590 & 7.125 & $1.73 \%$ & $1.89 \%$ \\
\hline $\operatorname{boxcox}_{1}$ & 6.140 & 4.032 & 5.231 & 4.958 & 4.244 & 5.372 & 4.946 & 1.802 & 5.845 & 5.987 & 4.589 & 7.074 & $1.28 \%$ & $1.08 \%$ \\
\hline boxcox 2 & 6.153 & 4.035 & 5.242 & 4.967 & 4.255 & 5.523 & 4.988 & 1.808 & 5.842 & 5.989 & 4.596 & 7.152 & $1.80 \%$ & $1.62 \%$ \\
\hline poly $_{1}$ & 6.113 & 4.016 & 5.211 & 4.923 & 4.234 & 5.284 & 4.939 & 1.798 & 5.854 & 6.001 & 4.579 & 7.066 & $0.93 \%$ & $0.60 \%$ \\
\hline poly $_{2}$ & 6.134 & 4.018 & 5.226 & 4.933 & 4.245 & 5.457 & 4.987 & 1.807 & 5.841 & 5.993 & 4.587 & 7.173 & $1.54 \%$ & $0.99 \%$ \\
\hline $\operatorname{mlog}{ }_{1}$ & 6.098 & 4.020 & 5.206 & 4.924 & 4.235 & 5.257 & 4.928 & 1.796 & 5.870 & 6.016 & 4.577 & 7.049 & $0.86 \%$ & $0.60 \%$ \\
\hline $\mathrm{mlog}_{2}$ & 6.118 & 4.023 & 5.220 & 4.928 & 4.246 & 5.419 & 4.976 & 1.803 & 5.850 & 6.001 & 4.584 & 7.158 & $1.42 \%$ & $0.86 \%$ \\
\hline N-PIT & 6.054 & 4.004 & 5.162 & 4.890 & 4.188 & 5.205 & 4.847 & 1.826 & 5.854 & 5.993 & 4.553 & 7.129 & $0.45 \%$ & $1.47 \%$ \\
\hline t-PIT & 6.154 & 4.089 & 5.197 & 4.961 & 4.264 & 5.264 & 4.901 & 1.868 & 5.824 & 5.984 & 4.611 & 6.991 & $1.37 \%$ & $1.13 \%$ \\
\hline
\end{tabular}

(respectively, 31 Dec 2012) are determined. Then the window is rolled forward by one day, the models are reestimated and forecasts for all 24 hours of the next day are computed. This procedure is repeated until the predictions for the 24 hours of 28 Jul 2016 (respectively, 17 Dec 2013) are computed. Note, that for the European datasets the out-of-sample test period covers roughly four years (1459 days) of data and for the GEFCom 2014 dataset with only 352 days.

\section{A. MAE, RMSE and m.p.d.f.b error measures}

As the main evaluation criterion we consider the Mean Absolute Error (MAE) for the full out-of-sample test period of $D=1459$ days (for GEFCom2014 only 352 days). It is computed for each VST and dataset as:

$$
\text { MAE }=\frac{1}{24 D} \sum_{d=1}^{D} \sum_{h=1}^{24}\left|\widehat{\varepsilon}_{d, h}\right|,
$$

where $\widehat{\varepsilon}_{d, h}$ denotes the estimated forecasting error for day $d$ and hour $h$. The MAE errors are reported for the 16 considered VSTs and all 12 datasets in Table II. We have also analyzed Root Mean Square Errors (RMSE):

$$
\operatorname{RMSE}=\sqrt{\frac{1}{24 D} \sum_{d=1}^{D} \sum_{h=1}^{24} \widehat{\varepsilon}_{d, h}^{2},}
$$

but the results were similar and hence are not reported nor analyzed here due to space limitations (but are available from the authors upon request). Only in Table II we provide for comparison an aggregate measure of fit based on the RMSE - the m.p.d.f.b. - as defined in (18) below. Although there are some changes in the ranking, the overall picture is very similar.
Given the large number of results it is hard to rank the VSTs. To tackle this issue, following [13], we introduce the mean percentage deviation from the best (m.p.d.f.b.) VST, which is inspired by the m.d.f.b. measure used in [11], [28] for comparing models. The m.p.d.f.b. measure for VST $i$ indicates how similar is this VST's performance to the 'optimal VST' composed of the best performing VST for each of the 12 datasets:

$$
\text { m.p.d.f.b. }{ }_{i}=\frac{1}{12} \sum_{j=1}^{12} \frac{\left|\mathrm{ERR}_{i, j}-\mathrm{ERR}_{\text {best VST }, j}\right|}{\operatorname{ERR}_{\text {best VST }, j}} \times 100 \%,
$$

where $\mathrm{ERR}_{\text {best VST }, j}=\min _{1 \leq i \leq 17} \mathrm{ERR}_{i, j}$ and ERR can be the MAE, the RMSE or any other error measure for point forecasts. The m.p.d.f.b. measure is reported for the original data and 16 considered VSTs in the last two columns of Table II.

From Table II we can see the dominance of the N-PIT over the competitors in terms of MAE. It has the lowest m.p.d.f.b., nearly twice smaller than the next best transform, i.e., the $\mathbf{m} \log _{1}$. Also for four datasets (EPEX.DE+AT, EXAA.DE+AT, NP.DK2 and OTE.CZ) it is the best performer and second best for another two (BELPEX.BE and NP.DK1). However, for some markets (NP.SYS, OMIE.ES and GEFCom2014) it does not excel. The t-PIT transformation is the best for three markets (OMIE.ES, OMIE.PT and GEFCom2014), but the overall performance does not seem to be robust and the tPIT forecasting accuracy is poor for some markets (especially EPEX.CH and NP.SYS). The $\mathbf{3} \sigma, \mathbf{3} \sigma \log$ and asinh transformations show moderate MAE forecasting performance. Still, their m.p.d.f.b. is better than that of the original (i.e, untransformed) data and sometimes even that of the best transformation for a given market ( $3 \sigma_{1}$ for EPEX.CH, $3 \sigma_{2}$ for EPEX.FR and $\mathbf{a s i n h}_{1}$ for NP.DK1). 
In terms of RMSE, both N-PIT and t-PIT are on average outperformed by the generalizations of the Box-Cox transform, i.e., poly and mlog, which show in general a very similar behavior across all 12 datasets. The boxcox and PIT transformations follow closely, while the remaining ones lag behind. Interestingly, the t-PIT has a better RMSE forecasting accuracy than the N-PIT. Furthermore, we see that the logistic transformations are the only ones which are worse in terms of m.p.d.f.b. (both MAE and RMSE-based) than the original (i.e., untransformed) data. Still, somewhat surprisingly logistic l $_{2}$ is the best choice in terms of MAE for the Belgian market, which nicely illustrates that the overall behavior can depend on the considered market and data structure. Finally, we want to remark that there is no clear tendency if a scaling by set $_{1}$ (median, MAD) or set $_{2}$ (mean, std) is preferable, although for the Box-Cox type transformations (boxcox, poly and mlog) set $_{1}$ leads to marginally better predictions in terms of m.p.d.f.b., see Table II.

\section{B. Diebold-Mariano tests}

The MAE values analyzed in Section IV-A can be used to provide a ranking of transformations, but not statistically significant conclusions on the outperformance of the forecasts of one transformation by those of another. Therefore, we also computed the Diebold-Mariano (DM) test [14], which takes the correlation structure into account. It tests forecasts of each pair of transformations against each other.

In the EPF literature, the DM test is usually performed separately for each of the 24 hours of the day [1]. However, Ziel and Weron [13] recently introduced a different approach, where only one statistic for each pair of models (here: VSTs) is computed based on the 24-dimensional vector of errors for each day, and called it the multivariate or vectorized DM test. Following [13], denote by $\widehat{\varepsilon}_{X, d}=\left(\widehat{\varepsilon}_{X, d, 1}, \ldots, \widehat{\varepsilon}_{X, d, 24}\right)^{\prime}$ and $\widehat{\varepsilon}_{Y, d}=\left(\widehat{\varepsilon}_{Y, d, 1}, \ldots, \widehat{\varepsilon}_{Y, d, 24}\right)^{\prime}$ the vectors of out-of-sample errors for day $d$ of VSTs $X$ and $Y$, respectively. Then the multivariate loss differential series:

$$
\Delta_{X, Y, d}=\left\|\widehat{\varepsilon}_{X, d}\right\|_{p}-\left\|\widehat{\varepsilon}_{Y, d}\right\|_{p},
$$

defines the differences of errors in the $\|\cdot\|_{p}$-norm, i.e., $\left\|\widehat{\varepsilon}_{X, d}\right\|_{p}=\left(\sum_{h=1}^{24}\left|\widehat{\varepsilon}_{X, d, h}\right|^{p}\right)^{1 / p}$ for $p=1,2$. For each model pair and each dataset we compute the $p$-value of two one-sided DM tests: (i) a test with the null hypothesis $H_{0}: E\left(\Delta_{X, Y, d}\right) \leq 0$, i.e., the outperformance of the forecasts of $Y$ by those of $X$, and (ii) the complementary test with the reverse null $H_{0}^{R}: E\left(\Delta_{X, Y, d}\right) \geq 0$, i.e., the outperformance of the forecasts of $X$ by those of $Y$. As in the standard DM test, we assume that the loss differential series is covariance stationary.

To jointly evaluate the performance of the VSTs across all 11 European markets we introduce yet another variant of the DM test, in which the norm is computed not only for all hours but all datasets as well. Note, that we exclude GEFCom 2014 from this analysis due to a much shorter test period. The computations are analogous, only this time the multivariate loss differential series across all 11 European markets, i.e., $M_{1}=$ BELPEX.BE, ..., $M_{11}=$ OTE.CZ, is given by:

$$
\Delta_{X, Y, d, M}=\left\|\widehat{\varepsilon}_{X, d}\right\|_{p}-\left\|\widehat{\varepsilon}_{Y, d}\right\|_{p},
$$

where now $\left\|\widehat{\varepsilon}_{X, d}\right\|_{p}=\left(\sum_{j=1}^{11} \sum_{h=1}^{24}\left|\widehat{\varepsilon}_{X, d, h, M_{j}}\right|^{p}\right)^{1 / p}$ and $\widehat{\varepsilon}_{X, d}=\left(\widehat{\varepsilon}_{X, d, 1, M_{1}}, \ldots, \widehat{\varepsilon}_{X, d, 24, M_{11}}\right)^{\prime}$ is the vector of out-ofsample errors all hours and all markets on day $d$.

In Figure 3 we plot the results for the multivariate DMtest for each market using the $\|\cdot\|_{1}$-norm, i.e., for $p=1$ in Eqn. (19), while in Figure 4 the aggregated DM-test as defined in Eqn. (20). In both figures we see the corresponding $p$-values of the conducted pairwise comparisons. Green and yellow squares indicate statistical significance at the $5 \%$ level. For instance, we see in Fig. 3 for BELPEX.BE that the first row is completely green. So every transformation significantly improved the forecasting accuracy compared to the original untransformed prices. Similarly for EXAA.DE+AT, the column which corresponds to the N-PIT is dark green, meaning that N-PIT leads to significantly better forecasts than all other transformations under consideration.

Regarding the aggregated DM-tests in Fig. 4, we see that all transformations lead to significantly better predictions than the original data, except for logistic. This result holds for the $\|\cdot\|_{1^{-}}$ and the $\|\cdot\|_{2}$-norm, even though the latter tends to return higher $p$-values. For the $\|\cdot\|_{1}$-norm the N-PIT transformation leads to significantly better forecasts than all other options, which emphasizes the findings from Table II. But for the $\|\cdot\|_{2}$-norm the results are not that clear-cut. Still poly $_{1}$ and $\mathbf{m l o g}_{1}$ lead to forecasts that outperform most of the competitors, except each other, $\mathbf{3} \boldsymbol{\sigma}_{\mathbf{2}}$ and N-PIT. Heaving this in mind, the N-PIT seems to be the overall best performer. It leads to significantly better predictions than all other transformations within the robust evaluation framework with respect to the $\|\cdot\|_{1}$-norm and not significantly worse than the best transformations in the $\|\cdot\|_{2^{-}}$ norm framework. However, if the evaluation focus is on spike detection then we suggest to use the $\operatorname{mog}_{1}$ as it has a very similar performance to the poly $_{1}$, but requires only one shape parameter (i.e., $c$ ).

\section{Conclusions}

We have conducted an extensive EPF study across 12 major markets to evaluate different variance stabilizing transformations. In line with the guidelines set forth in [1], we have used two variants of the Diebold-Mariano (DM) test to formally assess the statistical significance of the forecasting performance. The obtained results suggest that the choice of the optimal transformation depends on the forecasting framework and the considered dataset.

However, while for individual markets specifically tailored transformations can yield better results, due to the increasing demand for joint modeling of multiple markets, robust crossmarket transformations may turn out to be very useful. In particular, the probability integral transform-based N-PIT yields very robust results and promising forecasting accuracy in terms of MAE. It leads to forecasts that significantly outperform all other competitors across all markets, according to the $\|\cdot\|_{1^{-}}$ norm based DM test. However, if the forecasting focus is 

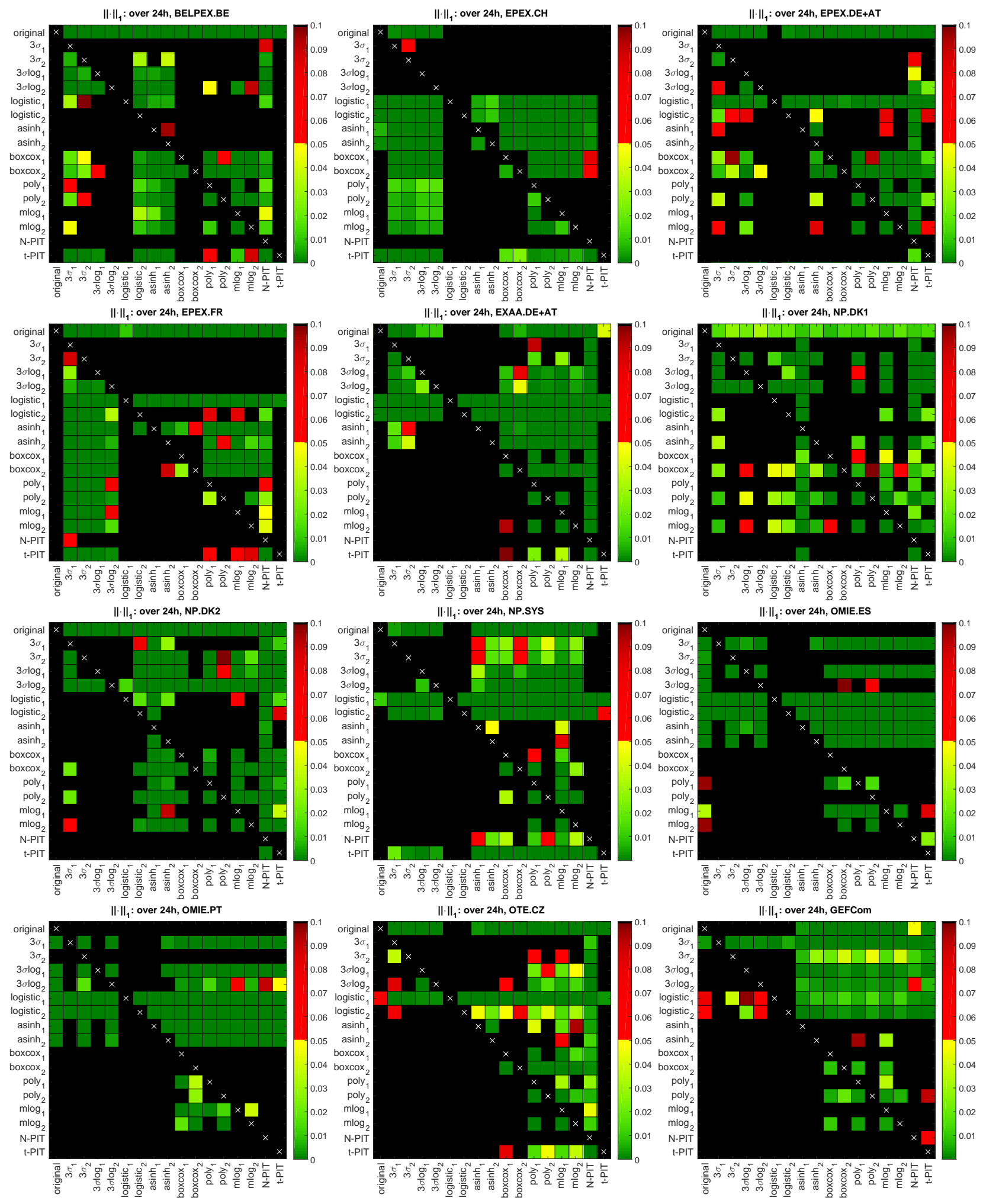

Fig. 3. Results of the 'multivariate' DM test defined by the multivariate loss differential series in Eqn. (19) with $p=1$, i.e., in the $\|\cdot\|_{1}$-norm, for all 12 datasets. Like in Figure 4, we use a heat map to indicate the range of the $p$-values - the closer they are to zero ( $\rightarrow$ dark green) the more significant is the difference between the forecasts of a model on the $\mathrm{X}$-axis (better) and the forecasts of a model on the Y-axis (worse). 

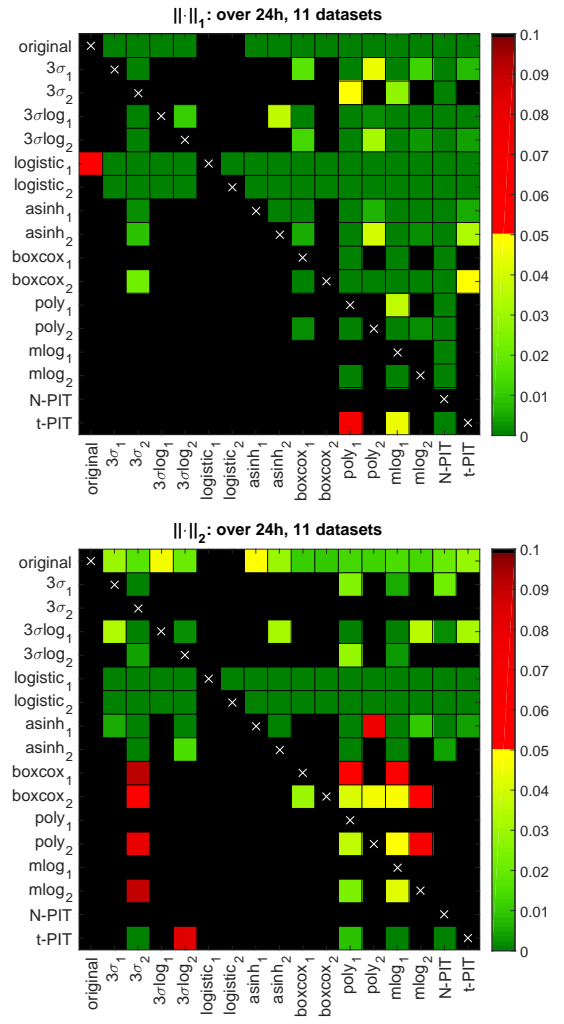

Fig. 4. Results of the 'multivariate' DM test defined by the multivariate loss differential series in Eqn. (20) with $p=1$ (top) and $p=2$ (bottom) across all 11 European datasets. We use a heat map to indicate the range of the $p$-values - the closer they are to zero $(\rightarrow$ dark green) the more significant is the difference between the forecasts of a model on the $\mathrm{X}$-axis (better) and the forecasts of a model on the Y-axis (worse).

on spike sensitive measures (like the RMSE), then the newly introduced poly and mlog transforms tend to perform better. Given that the mlog requires only one shape parameter $(c)$, while poly needs two $(\lambda, c)$, we suggest to use the former in such a context.

Our study can be further expanded in several directions. In particular, we report results for only one expert, regressionbased model. Although we have also considered several other expert and autoregressive models from [13], [15] and the results were qualitatively the same, we can only conjecture that our conclusions will hold for more complex models, like parameter rich structures estimated via the LASSO [13], [15], [19] or well performing neural network feature selection algorithms [29]. Moreover, in this study we have restricted ourselves to symmetric transformations. Future research could elaborate on asymmetric functions, which may yield an even better forecasting performance.

\section{REFERENCES}

[1] R. Weron, "Electricity price forecasting: A review of the state-of-theart with a look into the future," International Journal of Forecasting, vol. 30, no. 4, pp. 1030-1081, 2014.

[2] E. Fanone, A. Gamba, and M. Prokopczuk, "The case of negative dayahead electricity prices," Energy Economics, vol. 35, pp. 22-34, 2013.

[3] J. Janczura, S. Trück, R. Weron, and R. Wolff, "Identifying spikes and seasonal components in electricity spot price data: A guide to robust modeling," Energy Economics, vol. 38, pp. 96-110, 2013.

[4] P. J. Huber and E. M. Ronchetti, Robust Statistics, 2nd ed. Wiley, 2009.
[5] J. H. Zhao, Z. Y. Dong, X. Li, and K. P. Wong, "A framework for electricity price spike analysis with advanced data mining methods," IEEE Transactions on Power Systems, vol. 22, no. 1, pp. 376-385, 2007.

[6] R. Weron, "Heavy-tails and regime-switching in electricity prices," Mathematical Methods of Operations Research, vol. 69, no. 3, pp. 457473, 2009.

[7] D. Chen and D. W. Bunn, "Analysis of the nonlinear response of electricity prices to fundamental and strategic factors," IEEE Transactions on Power Systems, vol. 25, p. 595-606, 2010.

[8] T. Hong, P. Pinson, S. Fan, H. Zareipour, A. Troccoli, and R. J. Hyndman, "Probabilistic energy forecasting: Global Energy Forecasting Competition 2014 and beyond," International Journal of Forecasting, vol. 32, no. 3, pp. 896-913, 2016.

[9] M. Shahidehpour, H. Yamin, and Z. Li, Market Operations in Electric Power Systems: Forecasting, Scheduling, and Risk Management. Wiley, 2002.

[10] J. Contreras, R. Espínola, F. Nogales, and A. Conejo, "ARIMA models to predict next-day electricity prices," IEEE Transactions on Power Systems, vol. 18, no. 3, pp. 1014-1020, 2003.

[11] R. Weron and A. Misiorek, "Forecasting spot electricity prices: A comparison of parametric and semiparametric time series models," International Journal of Forecasting, vol. 24, pp. 744-763, 2008.

[12] S. Schneider, "Power spot price models with negative prices," Journal of Energy Markets, vol. 4, no. 4, pp. 77-102, 2011.

[13] F. Ziel and R. Weron, "Day-ahead electricity price forecasting with high-dimensional structures: Univariate vs. multivariate models," Energy Economics, 2017, submitted.

[14] F. X. Diebold and R. S. Mariano, "Comparing predictive accuracy," Journal of Business and Economic Statistics, vol. 13, pp. 253-263, 1995.

[15] B. Uniejewski, J. Nowotarski, and R. Weron, "Automated variable selection and shrinkage for day-ahead electricity price forecasting," Energies, vol. 9, no. 8, p. 621, 2016.

[16] M. Burger, B. Graeber, and G. Schindlmayr, Managing energy risk: An integrated view on power and other energy markets. Wiley, 2007.

[17] R. Weron, Modeling and Forecasting Electricity Loads and Prices: A Statistical Approach. John Wiley \& Sons, Chichester, 2006.

[18] N. Karakatsani and D. W. Bunn, "Forecasting electricity prices: The impact of fundamentals and time-varying coefficients," International Journal of Forecasting, vol. 24, pp. 764-785, 2008.

[19] F. Ziel, "Forecasting electricity spot prices using LASSO: On capturing the autoregressive intraday structure," IEEE Transactions on Power Systems, vol. 31, no. 6, pp. 4977-4987, 2016.

[20] J. S. Armstrong, Ed., Principles of Forecasting: A handbook for researchers and practitioners. Kluver, 2001.

[21] N. Hamdy, Applied Signal Processing: Concepts, Circuits, and Systems. CRC Press, Boca Raton, 2008.

[22] F. Günther and S. Fritsch, "neuralnet: Training of neural networks," The $R$ journal, vol. 2, no. 1, pp. 30-38, 2010.

[23] R. Hyndman and G. Athanasopoulos, Forecasting: Principles and practice. Online at http://otexts.org/fpp/, 2013.

[24] R. Sakia, "The Box-Cox transformation technique: A review," The Statistician, vol. 41, no. 2, pp. 169-178, 1992.

[25] T. Gneiting, F. Balabdaoui, and A. Raftery, "Probabilistic forecasts, calibration and sharpness," Journal of the Royal Statistical Society B, vol. 69, pp. 243-268, 2007.

[26] A. Der Kiureghian and P.-L. Liu, "Structural reliability under incomplete probability information," Journal of Engineering Mechanics, vol. 112, no. 1, pp. 85-104, 1986.

[27] G. Diaz and E. Planas, "A note on the normalization of Spanish electricity spot prices," IEEE Transactions on Power Systems, vol. 31, no. 3, pp. 2499-2500, 2016.

[28] J. Nowotarski, E. Raviv, S. Trück, and R. Weron, "An empirical comparison of alternate schemes for combining electricity spot price forecasts," Energy Economics, vol. 46, pp. 395-412, 2014.

[29] O. Abedinia, N. Amjady, and H. Zareipour, "A new feature selection technique for load and price forecast of electrical power systems," IEEE Transactions on Power Systems, vol. 32, no. 1, pp. 62-74, 2017. 


\section{HSC Research Report Series 2017}

For a complete list please visit http://ideas.repec.org/s/wuu/wpaper.html

01 Variance stabilizing transformations for electricity spot price forecasting by Bartosz Uniejewski, Rafał Weron and Florian Ziel 